Vascular Surgery

Elsevier Editorial System(tm) for Annals of

Manuscript Draft

Manuscript Number: AVS-D-19-00349R1

Title: Operative treatment and clinical outcomes in peripheral vascular trauma. The combined experience of two centres in the endovascular era.

Article Type: Basic Data

Corresponding Author: Dr. Ilenia D'Alessio, M.D.

Corresponding Author's Institution: Università degli studi di Milano

First Author: Ilenia D'Alessio, M.D.

Order of Authors: Ilenia D'Alessio, M.D.; Maurizio Domanin; Daniele Bissacco; Silvia Romagnoli; Pierantonio Rimoldi; Fabrizio Sammartano; Osvaldo Chiara

Abstract: Background. Arterial traumas of the extremities are quite rare in civilian records; nevertheless, patients with trauma of limbs are admitted daily in emergency departments worldwide. The up-to-date information about epidemiology and treatment (open vs endovascular surgery) comes from war records and it is not always easy getting data on mortality and morbidity in these patients. The aim of this study is to analyse the approach (open or endovascular) and the outcome of patients with vascular trauma of upper limbs (from the subclavian artery) and/or lower limbs (distal to the inguinal ligament), in the greater Milan area. Patients and methods. A retrospective analysis was conducted on data recorded by the emergency departments of two hospitals of the greater Milan between 2009-2017. We collected all patients with arterial injuries of the limbs in terms of demography, injury patterns, clinical status at admission, therapy (open or endovascular approach) and outcomes in terms of limb salvage and survival.

Results. We studied 52 patients with vascular trauma of extremities. The main mechanism of trauma was road accident (48.1\%), followed by criminal acts (32.7\%), self-endangering behaviour (13.5\%), work (3.8\%) and sport accidents (1.9\%). Associated lesions (orthopaedic, neurological and/or venous lesions of the limbs) were present in 39 patients (75\%). All patients underwent emergency surgery, forty six patients (88,5\%) by open repair (PTFE or greater saphenous vein bypass grafts, arterial suture or ligation) while endovascular approach was used only in 6 patients (11.5\%), all treated with embolization. The overall post-operative mortality rate was 5.7\% (3 patients). Among survivors, we report 5 major amputations of the lower limbs, 3 of them after bypass graft infection and 2 after graft failure. The rate of limbs salvage was $90.4 \%$. Conclusions Isolated arterial trauma of the extremities are rare, usually they occur in the setting of multiple trauma patients. Despite progresses in surgical techniques, there are still controversies in diagnosis and treatment of these patients. We treated the majority of cases with open surgery $(\mathrm{n}=46)$, choosing endovascular approach (embolization performed mainly by interventional radiologists) in difficult anatomic districts. We believe that, during decision making of the surgical strategy, it is important to consider the anatomical site of lesions and the general 
condition of the patients. Moreover, in case of multiple trauma, we suggest a multidisciplinary approach in order to provide the best medical care to the victims.

Suggested Reviewers: 
To T.M. Sullivan,

Editor-in-Chief

\section{Annals of Vascular Surgery}

\section{Dear Editor}

Please find enclosed the manuscript "Operative treatment and clinical outcomes in peripheral vascular trauma. The combined experience of two centres in the endovascular era." which we have revised, according to Reviewer's suggestions, and we would like to submit for publication in Annals of Vascular Surgery.

Our aim was to analyze retrospectively the treatment and the outcomes of vascular traumas of the extremities in the greater Milan area, between 2009-2017. We performed an analysis of data collected by two Milanese Institutes: ASST Ospedale Metropolitano Niguarda Ca' Granda and IRCCS Fondazione Ca' Granda Policlinico, the first of which with high flux trauma center.

Vascular injuries of upper and lower limbs are rare in civilian records, but despite their low epidemiologic impact, they could be life threatening lesions. We well know that the treatment of these lesions still remains a controversial filed. In the last years endovascular treatment is emerging and surely represents a valid approach, but we shouldn't forgot that often the majority of patients are multiple-injured and needed of emergency treatment for associated lesions. In this setting, the mini-invasive endovascular approach often remains confined to penetrating injuries in the treatment of areas with a difficult anatomic access or in injuries that can be treated not in an emergency setting.

All authors have read and approved the submitted manuscript and it has never been submitted nor published elsewhere in whole or in part. They do not have any conflict of interest in drugs, materials, or devices or financial disclosures and the study here presented does not violate any ethical principle.

We hope therefore you will consider our contribute for publication.

Sincerely yours

I.D'Alessio

M. Domanin

D. Bissacco

S. Romagnoli

P. Rimoldi

F. Sammartano

O. Chiara 
All correspondence can be addressed to

I.D’Alessio, (ilenia.dalessio@gmail.com)

Operative Unit of Vascular Surgery, Fondazione Ca' Granda Ospedale Maggiore Policlinico, Via Sforza 35, 20122 Milano, Italy. Tel. +39-02-55032438,

ilenia.dalessio@gmail.com 


\section{REVIEW OF THE PAPER}

\section{Ref. No.: AVS-D-19-00349}

Title: A two centers experience for extremity vascular trauma treatment: which is the real role of the endovascular repair?

\section{Dear Editor,}

The reviewers have recommended some revisions to our manuscript and we present our answer to their valuable questions.

\section{Reviewer 1}

English must be thoroughly revised because of many incorrect sentences and rough expressions

We have revised the entire manuscript, correcting typo and syntax errors.

Title: The title must definitely be modified because it doesn't reflect the content of the manuscript. One could expect that the main topic of the study is the role of endovascular therapy in the management of arterial trauma while the paper is much more descriptive and reporting global presentation and management of vascular trauma

We perfectly agree with Reviewer's observation and consequently we have changed the title trying to accord to the real object of this paper. The new title is: "Operative treatment and clinical outcomes in peripheral vascular trauma. The combined experience of two centres in the endovascular era"

Line 39-42 Abstract - Background: At the end of this subsection, the study objective must be stated

Done

Line 44 Abstract - Patients and Methods: "n=52" is a Result.

We agree. We have corrected this mistake

Line 46 Abstract - Patients and Methods: All the cited criteria are global items but no specific evaluation or judgement criterion is dedicated to endovascular treatement, as it would be expected when reading the title. Is this epidemiologic observational or comparative regarding two ways of managing trauma?

We agree with Reviewer's observation. The paper has an epidemiological-observational print. Our centers don't have preliminary criteria to assign patients to open surgery or embolization procedure. The decision making, in an emergency setting, is usually done considering the site of lesion and the general status of the patients.

Line 52-53 Abstract - Results: "largely" limited to embolization. This doesn't have a precise meaning.

According to Reviewer's observation we have rephrase that. 
Abstract - Conclusion: The conclusion doesn't match the objective and methods. "Covered stents" for example were never mentioned earlier,...etc

We agree and we thank for this observation. According to Reviewer's comment we have rewritten the conclusion in order to be consistent with the object of the paper

Line 82 Introduction: PTFE: The acronym must be explained when it is used for the first time

Done

Line 85-87 Introduction: The objective is not focusing on the role of endovascular techniques...

Thanks to reviewer's observations we have chosen to redraft some issues of this paper. It's more correct to state that our aim was given an epidemiological-observational view of our experience and not comparative study regarding two ways of managing trauma. Analyzing our experience we chosen to deepen the surgical approach that was used to treat our patient, including a focus on open and endovascular techniques

Line 91 Materials and methods: "52 patients" is a result. The number of included patients must be stated in the Results section.

Done

Line 95-96 Materials and methods: "Autochtonous or newly arrived immigrants". Is this truly relevant? Only if the aim of the paper is to describe social context of vascular trauma... Otherwise this criterion must be removed. Specially that the corresponding result is not cited further..

We agree and we apologize for this typo. It was included in the first draft of this paper with the aim of describing a social context, but we have decided to not include this analysis in the final version.

Line 118 Materials and methods: "Tab 1" is a Result and must be cited in the Results section.

Done

Line 107-109 Materials and methods: In my opinion the description of the statistical analysis is dispensable because the analyzed and compared outcomes are very few: Only one value is analyzed in all the manuscript, which is the association of lesions. It is not worth describing and performing the student test. 
We agree. We have corrected according to reviewer's suggestion.

Line 122 Results: Succession of many numbers and percentages makes the paragraph difficult to read. It is better to incorporate to Figure1.

Done. We have re-elaborate the graphic in figure 1 and we have incorporated the data both in the figure and in the caption

Line 160-164 Results: Describe the initial lesions that lead to amputation or death.

Done

Line 234-237 Discussion: risk factors for amputation. Please do the same analysis of your personal data. Discuss those, in the light of similar literature

Done

The conclusion is fine, but not is not keeping with the majority of the manuscript.

According to the Reviewer's suggestions we re-discussed the manuscript's conclusions, trying to be more adherent to the aim of the paper.

Reviewer 2

Line 150-153 I think it's necessary to explain the reason of the most frequent option for the arterial reconstruction of the lower limb is a graft

Thank you for your interesting observation. We have performed more frequently graft reconstruction because we didn't have much healthy arterial tissue, for direct suture We know that termino-terminal suture is ideal in complete traumatic transections, but in many cases this approach was impossible. We also know that bypass, using an inverted saphenous vein is the best revascularization technique in case of extensive arterial injury but, unfortunately, the ipsilateral vein was often not available due to associated venous injury and the harvest of the contralateral would have lengthened the operating time in patients with critical impairment.

\section{Editor}

Please present your references according to Vancouver rules

Done. We have followed the instructions for authors oh your homepage 
Anyway, we would thank the Referees for having appreciated our work and for their remarkable suggestions and comments, which have been taken into account to improve the original paper.

We hope that the present form the paper may be considered for publication.

With our best regards,

Ilenia D'Alessio, on the behalf of all the authors.

Correspondence to: Ilenia D'Alessio, MD

ilenia.dalessio@gmail.com 
1 Operative treatment and clinical outcomes in peripheral vascular trauma. The 2 combined experience of two centres in the endovascular era.

5 Ilenia D’Alessio ${ }^{\mathrm{a}}$, Maurizio Domanin ${ }^{\mathrm{b}-\mathrm{c} *}$, Daniele Bissacco ${ }^{\mathrm{a}}$, Silvia Romagnoli ${ }^{\mathrm{c}}$, Pierantonio 6 Rimoldi $^{\mathrm{e}}$, Fabrizio Sammartano ${ }^{\mathrm{d}}$, Osvaldo Chiara ${ }^{\mathrm{d}}$

8 a School of Vascular Surgery, University of Milan, Italy

9 b Department of Clinical Sciences and Community Health, University of Milan, Italy

10 c Operative Unit of Vascular Surgery, Fondazione I.R.C.C.S. Cà Granda Ospedale Maggiore 11 Policlinico, Milano, Italy

12 d DEA-EAS, General and Trauma Surgery department, ASST Grande Ospedale Metropolitano 13 Niguarda, Milano, Italy.

e Cardio-thoraco-vascular department, ASST Grande Ospedale Metropolitano Niguarda, Milano, 15 Italy.

* The authors equally contributed to this study

Corresponding author:

Ilenia D'Alessio

School of Vascular Surgery University of Milan

Via Commenda 19, 20122 Milan, Italy

Disclosure The authors have no personal, financial, or institutional interest in any of the drugs, materials, or devices described in this article.

KEYWORDS vascular trauma, extremities, open surgery, endovascular surgery

\section{Words count 3547}


ABSTRACT

Background. Arterial traumas of the extremities are quite rare in civilian records; nevertheless, patients with trauma of limbs are admitted daily in emergency departments worldwide. The up-todate information about epidemiology and treatment (open vs endovascular surgery) comes from war records and it is not always easy getting data on mortality and morbidity in these patients. The aim of this study is to analyse the approach (open or endovascular) and the outcome of patients with vascular trauma of upper limbs (from the subclavian artery) and/or lower limbs (distal to the inguinal ligament), in the greater Milan area.

Patients and methods. A retrospective analysis was conducted on data recorded by the emergency departments of two hospitals of the greater Milan between 2009-2017. We collected all patients with arterial injuries of the limbs in terms of demography, injury patterns, clinical status at admission, therapy (open or endovascular approach) and outcomes in terms of limb salvage and survival.

Results. We studied 52 patients with vascular trauma of extremities. The main mechanism of trauma was road accident $(48.1 \%)$, followed by criminal acts $(32.7 \%)$, self-endangering behaviour (13.5\%), work (3.8\%) and sport accidents (1.9\%). Associated lesions (orthopaedic, neurological and/or venous lesions of the limbs) were present in 39 patients (75\%). All patients underwent emergency surgery, forty six patients $(88,5 \%)$ by open repair (PTFE or greater saphenous vein bypass grafts, arterial suture or ligation) while endovascular approach was used only in 6 patients (11.5\%), all treated with embolization. The overall post-operative mortality rate was $5.7 \%$ (3 patients). Among survivors, we report 5 major amputations of the lower limbs, 3 of them after bypass graft infection and 2 after graft failure. The rate of limbs salvage was $90.4 \%$.

Conclusions Isolated arterial trauma of the extremities are rare, usually they occur in the setting of multiple trauma patients. Despite progresses in surgical techniques, there are still controversies in diagnosis and treatment of these patients. We treated the majority of cases with open surgery $(n=46)$, choosing endovascular approach (embolization performed mainly by interventional radiologists) in difficult anatomic districts. We believe that, during decision making of the surgical strategy, it is important to consider the anatomical site of lesions and the general condition of the patients. Moreover, in case of multiple trauma, we suggest a multidisciplinary approach in order to provide the best medical care to the victims. 
Manuscript

INTRODUCTION

Arterial traumas of the upper and lower extremities represent 3\% [1] of all civilian trauma and

include both blunt and penetrating injuries. The former usually are caused by car or motorcycle accidents and are the most frequent in civilian records, the latter mainly being related to criminal acts. While the majority of isolated injuries from penetrating trauma are successfully treated, blunt trauma lesions have poorer outcomes particularly in polytraumatized patients [2].

Despite the benefits achieved with the introduction of computed tomographic angiography (CTA) in the diagnostic process [3] the choice of the best treatment option, still remains unclear. Since the beginning of vascular surgery, open surgery has been the only option in treating arterial trauma for many decades. Several approaches, from direct suture of the injured vessel to bypass grafting using inverted saphenous vein, polytetrafluoroethylene (PTFE) or Dacron, have been performed. Nevertheless, more data are needed to properly select the optimal management for patients with arterial trauma of the extremities [4].

The aim of this retrospective study is to analyse the management and outcome of peripheral arterial trauma, merging the experience of two Institutions of the metropolitan area of Milan, with particular focus on the role of techniques (open or endovascular) used in our experience.

\section{MATERIALS AND METHODS}

The present study enrolled all patients with arterial traumas of the upper and lower extremities admitted between January 1st, 2009 and December 31st, 2017 to level 1 trauma center ASST Ospedale Metropolitano Niguarda and to IRCCS Fondazione Cà Granda Policlinico, both located in Milan.

We have retrospectively collected data taken from emergency departments database of the two Institutions, reporting age, gender, trauma's mechanism and nature (criminal, self-inflicted, road, sport and work accidents), Glasgow Coma $(\operatorname{GCS}(S))$, heart rate $(\operatorname{HR}(S))$ and systolic blood pressure $(\mathrm{SBP}(\mathrm{S}))$ on the scene(S). Moreover, Emergency Department (ED) evaluation of Glasgow Coma scale $(\mathrm{GCS}(\mathrm{ED}))$, heart rate $(\mathrm{HR}(\mathrm{ED}))$ and systolic blood pressure (SBP(ED)) were analysed. Lactates, the need of emergency surgery, the presence of injuries in other anatomic districts (head, chest and abdomen), the association with orthopaedic, neurological and venous lesions of limbs, the 
Injury Severity Score (ISS), the total length of stay and outcomes were also evaluated. We limited our analysis only to the extremities, from subclavian artery for the upper limbs and from the inguinal ligament for the lower limbs. We excluded from our analysis vascular trauma of the neck, chest and abdomen, iatrogenic lesions, complete sections of limbs and chronic lesions, such as pseudo-aneurysm in drug-addicted patients. The ISS threshold to define major trauma was 15.

Statistical analysis was performed using JMP 11.2.0 (SAS Institute Cary, NC). Data were presented in a descriptive form. Ordinal and nominal variables were reported as patients' percentage. Continuous variables were reported as average \pm standard deviation

\section{RESULTS}

During the study period, 52 patients with vascular injuries of limbs, 45 males (86.6\%) and 7 females $(13.4 \%)$ with mean age $37 \pm 18$ years, were analysed. In addition, parameters reported in Tab. 1, have been taken into account.

Five types of causes of injuries were found: road (25 patients, 48\%), criminal (17 patients, 32,7\%), inflicted self-endangering behavior (7 patients, 13,4\%), work (2 patients, 3,8\%) and sport (1 patient, $1,9 \%)$ accidents.

We also examined traumas depending on the anatomical site. We had 29 patients (55.8\%) with lesions distal to common femoral artery, 20 patients (38.5\%) with lesions below subclavian artery and 3 patients $(5.7 \%)$ with involvement of both anatomic districts. The relationship between mechanism of injury and type of lesions (blunt or penetrating) are showed in Fig. 1. Penetrating trauma occurred more frequently in upper extremities $(55.0 \%)$, while blunt trauma resulted more common in lower extremities lesions $(55.0 \%)$.

Since the majority of patients had multiple traumas, we evaluated several clinical parameters as reported in Tab. 1 with the purpose of giving a complete clinical grading. The majority of patients needed emergency surgery for vascular lesions $(86.5 \%)$, while the remains underwent other surgical procedures according to damage control strategy.

Many patients reported concomitant lesions in other anatomic districts: 2 patients $(3.8 \%)$ suffered head injuries, in 14 (26.9\%) chest was involved, while 12 (23.0\%) presented abdominal injuries. We also have analysed the presence of orthopaedic, neurological and venous lesions of limbs (Tab. 2). Orthopaedic lesions were the most frequent injuries associated with the vascular ones both for upper and lower extremities (12 and 17 patients respectively, 59.2\% of the study population), while venous lesions occurred more frequently in lower extremities traumas compared to upper limbs (6 
vs 2 patients). Six patients (12.2\%) were affected by two types of lesion in the same limb, while 5 $(10.2 \%)$ experienced venous, neurological and orthopaedic lesions in the same limb.

Regarding the type of artery involved, we reported 3 (10.3\%) lesions of the common femoral artery, $9(31.0 \%)$ of the superficial femoral artery, $4(13.7 \%)$ of the profunda femoris artery, 6 of popliteal artery, $3(10.3 \%)$ of anterior tibial artery, $2(6.8 \%)$ of posterior tibial artery, 1(3.4\%) of peroneal artery and $1(3.4 \%)$ with concomitant involvement of the common femoral artery and anterior tibial artery.

In upper limb traumas, $3(15 \%)$ cases involved the axillary artery, $9(45 \%)$ the brachial artery, 5 $(25 \%)$ the radial artery, $1(5 \%)$ the ulnar artery and $2(10 \%)$ both ulnar and radial arteries.

Three patients reported lesions of the common femoral artery associated to lesion of axillary, brachial and radial arteries. (Tab 3).

All patient underwent diagnostic CTA before treatment, and among them 8 (15.3\%) had angiography. The majority of patients underwent open surgery ( $n=46 / 52,88.5 \%)$, while endovascular approach has been performed only in 6 patients (11.5\%).

In the group with lower limbs lesions we performed: 12 bypass grafts (41.3\%), 8 arterial sutures (27.5\%), 4 arterial ligations (13.7\%), 3 coil embolization (10.3\%), 1 arterial thrombectomy (3.4\%) and 1 thigh amputation (3.4\%). We performed more frequently graft reconstruction since direct suture was not possible due to unhealthy arterial tissue. PTFE bypass was the most used technique since the ipsilateral saphenous was often injured and the harvest of the contralateral saphenous vein would have required more operating time in patients in critical conditions. In the group with lesions of upper limbs we carried out 10 direct suture (50.0\%), 4 bypass grafts (20.0\%), 2 coil embolization (10.0\%), 1 arterial ligation (5.0\%), 1 amputation (5.0\%), 1 arterial thrombectomy (5.0\%). One patient $(5.0 \%)$ died before reaching operating room because of associated lesions. In the group of patients with injuries in both (upper and lower) limbs, multiple direct sutures have been performed, associated in 1 patient to embolization procedure (Tab. 4).

The total length of stay in the hospital was $34 \pm 31$ days because the majority of patients suffered multiple traumas. The overall post-operative mortality rate was $5.7 \%$ (3 patients). Two patients died because of Multiple Organ Failure, 1 died because of cerebral haemorrhage in the first week after limb revascularization. Among survivors, we reported 5 major amputations of the lower limbs, 1 in the operating room, 2 after bypass infections and 2 after graft failure, 7 days and 32 days respectively after the first attempt of limb salvage.

\section{DISCUSSION}


Arterial traumas of the peripheral arteries of the extremities are a rare observation in patients admitted to the emergency department. Among civilians, ER evaluations are prevalently related to road accidents, criminal attacks and, less frequently, to self-harming behaviours, work and or sport traumatisms. [2,5]

As stated above, most patients with blunt trauma were poly-traumatized. In these cases, patients' life is the first priority, followed by limb salvage. The outcome in these patients is strictly influenced by co-morbidities and associated lesions. Multiple trauma patients are expected to present a higher risk of mortality than those with single injuries, being the overall mortality of multiple lesions significantly higher than mortality rates of single lesions. [6] Literature reports an association with bony injury in $35.1 \%$ of cases, nerve injury in $7.6 \%$, and injuries affecting the head, chest, or abdomen in 3.6\%. [7]

Regarding vascular injuries of limbs, it is important to differentiate minor arterial trauma, due to low speed agents, from major arterial trauma. In the first case, neither active bleeding is detected at CTA scan nor ischemia at physical examination. Therapeutic chooses usually consists in monitoring the patient during follow up, by means of physical examination and ultrasound scan. In case of major arterial trauma, with active bleeding and/or ischemia, the patient needs to be treated in the emergency room with basic and advanced life support.

Diagnostic imaging provides a valid support in identifying vascular injuries. Nowadays, delays in diagnosis and treatment are usually uncommon in patients with multiple injuries, due to the introduction of total-body CT scan, which allows a complete screening of severely injured patients with blunt multiple-trauma, determining whether surgical or angiographic intervention is needed. [7-8]

After diagnosis, a critical aspect in managing vascular injuries of limbs is constituted by treatment decision-making process.

Data regarding the management of complex extremity trauma lesions are conflicting and randomized controlled studies are not reported. The absence of Level 1 and 2 evidence studies is not surprising because of the complexity of the topic. In fact, there are frequently concomitant injuries, multiple traumas and heterogeneity of treatment. Moreover, the relative rarity of such lesions contributes to complicate the analysis and to reach a consensus on their treatment.

For decades the classic approach to vascular lesions of extremities has been open surgery, though the growing interest in endovascular treatment has developed in the last years. [9-10]

Piffaretti et al [11], reported treatment of lesions amenable to endovascular repair in 10 cases over $81(12.0 \%)$ of arterial trauma lesions, represented mainly by pseudoaneurysms, dissections and 
arterio-venous fistulas with only one case of expanding hematoma. Immediate success was obtained in all procedures.

Desai et al [12], in a 8 years retrospective analysis, reported 28 endovascular repair in 21 (75.0\%) penetrating injuries and $7(25.0 \%)$ blunt trauma including pseudoaneurysm, extravasations, occlusions and arteriovenous fistulas, without any further distinction about localization. Patients were selected for endovascular treatment in case of no pulsatile bleeding from the wound and a suitable access site with a lesion amenable to stent graft repair at preoperative imaging. They reported a $100 \%$ of immediate technical success with the use of covered stent, a mean length of stay of $18 \pm 22.9$ days. The overall limb salvage rate was $92 \%$ at 45 days and $79 \%$ at 93 days.

Ganapathy et al [4] collected 68 patients with 70 total arterial injuries. Endovascular approach was performed in 20 patients. Compared to open repair $(n=50)$, endovascular less commonly required fasciotomy ( $15 \%$ vs. $46 \%, \mathrm{p}=0.03)$ and transfusion (50\% vs. $77 \%, \mathrm{p}=0.06)$. Analysed outcomes between groups were trending higher in the endovascular group with respect to limb salvage rates at discharge (94\% vs. 89\%), median length of stay (14days vs. 9), and median follow-up (288days vs. 92) compared to the open group, but the data were not statistically significant. There was increasing utilization of endovascular repair over time (7\% of total procedures in 2009; $50 \%$ in 2014).

Tresson et al [13] reported endovascular treatment limited to the embolization of pelvic arteries in a series of 20 patients injured after Paris Terrorist Attack in 2015. They did not report the use of covered stents because of the devastating wounds associated, requiring always a surgical exploration.

In our cohort, endovascular treatment has been limited to 6 patients (11.5\%). Nearly $80 \%$ of our patients were either hemodynamically unstable or had multiple vessels injured on arrival to the ER. These conditions have made endovascular repair less suitable, limiting its use only in case of pseudoaneurysms [14] and/or arteriovenous fistulas, though rather uncommon in civilian trauma patients.

Despite all efforts, the prognosis of patients with arterial trauma is influenced by several factors, first and foremost by the degree of general impairment evaluated using ISS; second by the kind of trauma. Blunt trauma has a poorest prognosis compared to penetrating injuries that have an amputation rate three times greater. [15]. In our cohort, we have reported 6 cases of amputation, 5 among survivors and 1 in a deceased patient, with, in most cases, lesions of the popliteal artery. Furthermore, independent risk factors for amputation, including occluded graft, combined aboveand below-knee injury, compartmental syndrome, arterial transection, lack of intra or immediately postoperative anticoagulation and associated compound fracture, play a fundamental role in the clinical course[5,16]. 
237 In our study, as reported previously (see: Results section), we had 5 major amputations of the lower 238 limbs: 1 in the OR (operating room) for a devastating lesion above the popliteal artery, 2 after graft 239 infections with septic embolism and 2 after bypass failure for the lack of postoperative 240 anticoagulation because of concomitant brain bleeding.

\section{CONCLUSIONS}

In our experience, surgical treatment of peripheral vascular injuries is always a challenging issue, especially in complicated multiple trauma patients.

Although we are all aware of the revolutionary impact of the endovascular approach in many fields, open surgery still has a role in the management of vascular trauma of the extremities mainly in patients with severe concomitant lesions. In these cases, the high frequency of devastating injuries and the frequent need for orthopaedic and/or neurological repair, make endovascular surgery scarcely suitable. On the contrary, endovascular surgery may result fundamental for the treatment of penetrating injuries in areas of difficult anatomic access, such as the abdominal and pelvic area, the shoulder and the neck or, in case of more peripheral lesions whose treatment can be postponed [11,17]. To avoid any misleading generalization on the issue of arterial trauma repair, we strongly believe that, for each patient, it is necessary to consider the anatomical locations of the vascular injury and the degree of general impairment. Moreover, a multidisciplinary approach is essential in treating these complex patients. Cooperation among different surgical specialists (vascular, orthopaedic, thoracic and neurosurgeons) and interventional radiologists is the key to obtain the best clinical results. 


\section{REFERENCES}

[1] Compton C. Peripheral Vascular Trauma. Perspect Vasc Surg Endovasc Ther 2005;17:297-307.

[2] Martin LC, McKenney MG, Sosa JL, Ginzburg E, Puente I, Sleeman D, et al. Management of lower extremity arterial trauma. J Trauma 1994;37:591-8.

[3] Miller-Thomas MM, West OC, Cohen AM. Diagnosing Traumatic Arterial Injury in the Extremities with CT Angiography: Pearls and Pitfalls. RadioGraphics 2005;25:S133-42.

[4] Ganapathy A, Khouqeer AF, Todd SR, Mills JL, Gilani R. Endovascular management for peripheral arterial trauma: The new norm? Injury 2017;48:1025-30.

[5] Hafez HM, Woolgar J, Robbs J V. Lower extremity arterial injury: results of 550 cases and review of risk factors associated with limb loss. J Vasc Surg 2001;33:1212-9.

[6] Rau C-S, Wu S-C, Kuo P-J, Chen Y-C, Chien P-C, Hsieh H-Y, et al. Polytrauma Defined by the New Berlin Definition: A Validation Test Based on Propensity-Score Matching Approach. Int J Environ Res Public Health 2017;14:1045.

[7] Scaglione M, Pinto A, Pedrosa I, Sparano A, Romano L. Multi-detector row computed tomography and blunt chest trauma. Eur J Radiol 2008;65:377-88.

[8] Dreizin D, Munera F. Blunt Polytrauma: Evaluation with 64-Section Whole-Body CT Angiography. RadioGraphics 2012;32:609-31.

[9] Reuben BC, Whitten MG, Sarfati M, Kraiss LW. Increasing use of endovascular therapy in acute arterial injuries: Analysis of the National Trauma Data Bank. J Vasc Surg 2007;46:1222-6.

[10] Worni M, Scarborough JE, Gandhi M, Pietrobon R, Shortell CK. Use of Endovascular Therapy for Peripheral Arterial Lesions: An Analysis of the National Trauma Data Bank From 2007 to 2009. Ann Vasc Surg 2013;27:299-305.

[11] Piffaretti G, Tozzi M, Lomazzi C, Rivolta N, Caronno R, Laganà D, et al. Endovascular treatment for traumatic injuries of the peripheral arteries following blunt trauma. Injury 2007;38:1091-7.

[12] Desai SS, DuBose JJ, Parham CS, Charlton-Ouw KM, Valdes J, Estrera AL, et al. Outcomes after endovascular repair of arterial trauma. J Vasc Surg 2014;60:1309-14. 
[13] Tresson P, Touma J, Gaudric J, Pellenc Q, Le Roux M, Pierret C, et al. Management of Vascular Trauma during the Paris Terrorist Attack of November 13, 2015. Ann Vasc Surg 2017;40:44-9.

[14] Domanin M, Castronovo LE, Fossati A, Romagnoli S, Gabrielli L. Endovascular management of post-traumatic peroneal pseudoaneurysm associated to arteriovenous fistula after sport-related injury. J Traumatol du Sport 2017;34:114-8.

[15] Rozycki GS, Tremblay LN, Feliciano D V., McClelland WB. Blunt Vascular Trauma in the Extremity: Diagnosis, Management, and Outcome. J Trauma Inj Infect Crit Care 2003;55:814-24.

[16] Guerrero A, Gibson K, Kralovich KA, Pipinos I, Agnostopolous P, Carter Y, et al. Limb loss following lower extremity arterial trauma: What can be done proactively? Injury 2002;33:765-9.

[17] Urgnani F, Lerut P, Da Rocha M, Adriani D, Leon F, Riambau V. Endovascular treatment of acute traumatic thoracic aortic injuries: A retrospective analysis of 20 cases. J Thorac Cardiovasc Surg 2009;138:1129-38. 
Table's legend

Tab. 1. Parameters on the scene and at the arrival in the Emergency Department. SBPs= systolic 355 blood pressure on the scene of trauma. HRs=heart rate on the scene of trauma. GCSs=Glasgow coma scale on the scene of trauma. SPBed= systolic blood pressure at the arrival at emergency department. HRed= heart rate at the arrival at emergency department. GCSs= Glasgow Coma Scale at the arrival at emergency department. Lactates=lactates at the arrival at emergency department. ISS=Injury Severity Score.

\section{Tab. 2. Associated lesions related to localization of vascular injury at lower, upper or both limbs} $\mathrm{N}=$ number of patients.

Tab. 3. Detailed analysis of the arteries injured related to localization at lower, upper or both limbs. $\mathrm{N}=$ number of patient.

Tab. 4. Kind of treatment related to localization of vascular injury (lower, upper or both limbs) $\mathrm{N}=$ number of patient.

Figure's legend

Figure 1 Relationship between mechanism of injuries and localization at upper, lower or both limbs: $\mathrm{W}=$ work accidents, $\mathrm{C}=$ criminal acts, $\mathrm{R}=$ road accidents, $\mathrm{A}=$ self-inflicted acts, $\mathrm{S}=$ sport accidents. Among patients with lesions in the lower limbs we reported 16 R (55.2\%), 7 C (24.1\%) 4 A (13.8\%), $1 \mathrm{~W}(3.4 \%)$ and $1 \mathrm{~S}(3.4 \%)$. Among patients with lesions distal to the subclavian artery we reported $7 \mathrm{R}(35.0 \%), 9 \mathrm{C}(45.0 \%), 3 \mathrm{~A}(15.0 \%)$ and $1 \mathrm{~W}(5.0 \%)$. In the group of patients with involvement of both locations (upper and lower limbs) we reported $2 \mathrm{R}$ and $1 \mathrm{C}$. 
1 Operative treatment and clinical outcomes in peripheral vascular trauma. The

2 combined experience of two centres in the endovascular era.

A two-centers experience for extremity vascular trauma treatment: which is the

4 real role of the endovascular repair?

5

6

7 Ilenia D’Alessio ${ }^{\mathrm{a}}$, Maurizio Domanin ${ }^{\mathrm{b}-\mathrm{c} *}$, Daniele Bissacco ${ }^{\mathrm{a}}$, Silvia Romagnoli ${ }^{\mathrm{c}}$, Pierantonio

8 Rimoldi $^{\mathrm{e}}$, Fabrizio Sammartano ${ }^{\mathrm{d}}$, Osvaldo Chiara ${ }^{\mathrm{d}}$

9

10 a School of Vascular Surgery, University of Milan, Italy

11 b Department of Clinical Sciences and Community Health, University of Milan, Italy

12 c Operative Unit of Vascular Surgery, Fondazione I.R.C.C.S. Cà Granda Ospedale Maggiore 13 Policlinico, Milano, Italy

14 d DEA-EAS, General and Trauma Surgery department, ASST Grande Ospedale Metropolitano 15 Niguarda, Milano, Italy.

16 e Cardio-thoraco-vascular department, ASST Grande Ospedale Metropolitano Niguarda, Milano, 17 Italy.

" The authors equally contributed to this study

Corresponding author:

Ilenia D'Alessio

School of Vascular Surgery University of Milan

Via Commenda 19, 20122 Milan, Italy

Disclosure The authors have no personal, financial, or institutional interest in any of the drugs, materials, or devices described in this article.

KEYWORDS vascular trauma, extremities, open surgery, endovascular surgery 
Background. Arterial traumas of the extremities are quite rare in civilian records, nevertheless patients with trauma of limbs are admitted daily in emergency departments worldwide. The up-todate information about epidemiology and treatment (open vs endovascular surgery) comes from war records and it is not always easy getting data on mortality and morbidity in these patients. The aim of this study is to analyse approach (open or endovascular) and the outcome of patients with vascular trauma of upper limbs (from the subclavian artery) and/or lower limbs (distal to the inguinal ligament), in the greater Milan area.

Patients and methods. A retrospective analysis was conducted on data recorded by the emergency departments of two hospitals of the greater Milan between 2009-2017. We studied all patients ( $\mathrm{n}=$ 52) with arterial injuries of the limbs in terms of demography, injury patterns, clinical status at admission, therapy (open or endovascular approach) and outcomes in terms of limb salvage and survival.

Results. We studied 52 patients with vascular trauma of extremities. The main principal mechanism of trauma was road accident (48.1\%), followed by criminal acts $(32.7 \%)$, self-inflicted lesions endangering behavior related trammas (13.5\%), work (3.8\%) and sport accidents (1.9\%). Associated lesions (orthopedic, neurological and/or superficial venous lesions of the limbs) were present in 39 patients (75\%). All patients were operated- underwent emergency surgery, forty six patients $(88,5 \%)$ by open repair (PTFE or greater saphenous vein bypass grafts, arterial suture or ligation) while endovascular approach was used only in 6 patients $(11.5 \%)$, all treated-with embolization. procedure and was largely limited to embolization.

The overall post-operative mortality rate was 5.7\% (3 patients). Among survivors, we report 5 major amputations of the lower limbs, 3 of them after bypass graft infection and 2 after graft failure. The overall mortality rate was $5.8 \%$, The rate of limbs salvage was $90.4 \%$.

Conclusions Isolated arterial trauma of the extremities are rare, usually they occur in the setting of multiple trauma patients. Despite progresses in surgical techniques, there are still controversies in diagnosis and treatment of these patients. We treated the majority of cases with open surgery ( $n=46)$, choosing to resort to endovascular treatment approach (embolization performed mainly by interventional radiologists) in all cases of single vascular lesions; this approach is useful in difficult anatomic districts. Covered stents are mostly not suitable to treat polytraumatized patients with associated wounds requiring a surgical exploration. We believe that, during decision making of the surgical strategy, it is important to consider the anatomical site of lesions and the general condition of the patients. Moreover, in case of multiple trauma, we suggest a multidisciplinary approach in order to provide the best medical care to the victims. 


\section{Manuscript}

\section{INTRODUCTION}

Arterial traumas of the upper and lower extremities represent 3\% [1] of all civilian trauma and include either blunt and penetrating injuries. The former usually are caused by car or motorcycle accidents and are the most frequent in civilian records, being the latter mainly being related to criminal acts. While the majority of isolated injuries from penetrating trauma are successfully treated, blunt trauma lesions have poorer outcomes particularly in polytraumatized patients.[2]' Despite the benefits achieved with the introduction of computed tomographic angiography (CTA) in the diagnostic process[3] ${ }^{2}$ the choice of the best treatment option, still remains unclear. Since the beginning of vascular surgery, open surgery has been the only option in treating arterial trauma preferred therapeutic option for the treatment of arterial trauma for many decades. Several approaches, from direct suture of the injured vessel to bypass grafting using inverted saphenous vein, polytetrafluoroethylene (PTFE) or Dacron, have been performed. Nevertheless, more data are needed to properly select the optimal management for patients with arterial trauma of the extremities.[4]

The aim of this retrospective study analysis is to evaluate analyse the management and outcome of peripheral arterial trauma incidence and the short term results of the surgical interventions performed for arterial traumas of the extremities, merging the experience of two Institutions of the metropolitan area of Milan, with particular focus on the role of techniques (open or endovascular) used in our experience.

\section{MATERIALS AND METHODS}

The present study enrolled all 52 patients with arterial traumas of the upper and lower extremities admitted between January 1st, 2009 and December 31st, 2017 to tertiary level 1 trauma center ASST Ospedale Metropolitano Niguarda and to IRCCS Fondazione Cà Granda Policlinico, both located in Milan.

We have retrospectively collected data taken from emergency departments database of the two Institutions, reporting age, gender, autochthønous or newly arrived immigrants from European or extra-European countries, the trauma's mechanism and nature (criminal, self-inflicted, road, sport and work accidents), Glasgow Coma $(\mathrm{GCS}(\mathrm{S}))$, heart rate $(\mathrm{HR}(\mathrm{S}))$ and systolic blood pressure $(\mathrm{SBP}(\mathrm{S}))$ on the scene(S). Moreover, Emergency Department (ED) evaluation of Glasgow Coma 
scale (GCS(ED)), heart rate (HR(ED)) and systolic blood pressure (SBP(ED)) were analysed. Lactates, the need of emergency surgery, the surgery choice, the presence of injuries in other anatomic districts (head, chest, and abdomen), the association with orthopedic, neurological and venous lesions of limbs, the Injury Severity Score (ISS), the total length of stay and elinical and strgicat outcomes were also evaluated. (Tab 1)

We limited our analysis only to the extremities, whose threshold was the distal segment of the from subclavian artery for the upper limbs and the inguinal ligament for the lower limbs. We excluded from our analysis vascular trauma of the neck, chest and abdomen, iatrogenic lesions, complete sections of limbs total traumatic amputation and chronic lesions, such as pseudo-aneurysm in drugaddicted patients. The ISS threshold to define major trauma was 15.

Statistical analysis was performed using JMP 11.2.0 (SAS Institute Cary, NC). Student t test was used to rate statistically significant differences between two independent means of sampled data. Data were presented in a descriptive form. Ordinal and nominal variables were reported as patients' percentage. Continuous variables were reported as average \pm standard deviation

\section{RESULTS}

During the study period, 52 patients with vascular injuries of limbs, 45 males (86.6\%) and 7 females $(13.4 \%)$ with mean age $37 \pm 18$ years, were analysed. In addition, parameters reported in Tab. 1, have been taken into account.

Five types of causes of injuries were found: road (25 patients, 48\%), criminal (17 patients, 32,7\%), inflicted self-endangering behavior ( 7 patients, 13,4\%), work (2 patients, 3,8\%) and sport (1 patient, $1,9 \%)$ accidents.

We also examined traumas depending on the anatomical site. We had 29 patients (55.8\%) with lesions distal to common femoral artery, 20 patients (38.5\%) with lesions below subclavian artery and 3 patients $(5.7 \%)$ with involvement of both anatomic districts. The relationship between mechanism of injury and type of lesions are showed in Fig. 1. Among patients with lesions in the lower limbs we reported 16 road accidents (55.2\%), 7 criminal acts (24.1\%) 4 self inflicted acts (13.8\%), 1 work accident (3.4\%) and 1 sport accident (3.4\%). Among patients with lesions distal to the subclavian artery we reported 7 roal accidents $(35.0 \%)$, 9 eriminal acts (45.0\%), 3 self inflicted acts (15.0\%) and 1 work accident (5.0\%). In the group of patients with involvement of both locations (upper and lower limbs) we reported 2 road accidents and 1 criminal act. (Fig 1 )

Penetrating trauma occurred more frequently in among upper extremities lesions (55.0\%), while blunt trauma resulted more common in lower extremities lesions occurred $(55.0 \%)$. ( $\operatorname{Tab} 2)$ 
Since the majority of patients had multiple traumas poly trammatized, we have evaluated several clinical parameters as reported in tab 1 with the purpose of giving a complete clinical grading. The majority of patients needed emergency surgery for vascular lesions (86.5\%), while the remains underwent other surgical procedures according to damage control strategy.

Many patients reported concomitant lesions in other anatomic districts: 2 patients (3.8\%) suffered head injuries, in 14 (26.9\%) chest was involved and, among them, 6 patients reported two-different kind of chest-lesions, while $12(23.0 \%)$ presented abdominal injuries, 5 - of whose reported two or more abdominal lesions.

We also have analysed the presence of orthopedic, neurological and venous lesions of limbs (Tab. 2 ). Orthopedic lesions were the most frequent injuries associated with the vascular ones both for upper and lower extremities (12 and 17 patients respectively, 59.2\% of the study population), while venous lesions occurred more frequently in lower extremities traumas compared to upper limbs (6 vs 2 patients). Six patients (12.2\%) were affected by two types of lesion in the same limb, while 5 (10.2\%) experienced all venous, neurological and orthopedic lesions in the same limb.

Regarding the type of artery involved, we reported $3(10.3 \%)$ lesions of the common femoral artery, $9(31.0 \%)$ of the superficial femoral artery, $4(13.7 \%)$ of the profunda femoris artery, 6 of popliteal artery, $3(10.3 \%)$ of anterior tibial artery, $2(6.8 \%)$ of posterior tibial artery, 1(3.4\%) of peroneal artery and $1(3.4 \%)$ with concomitant involvement of the common femoral artery and anterior tibial artery.

In upper limb traumas, $3(15 \%)$ cases involved the axillary artery, 9 (45\%) the brachial artery, 5 $(25 \%)$ the radial artery, $1(5 \%)$ the ulnar artery and $2(10 \%)$ both ulnar and radial arteries.

Three patients reported lesions of the common femoral artery associated to lesion of axillary, brachial and radial arteries. (Tab 3).

All patient underwent diagnostic CTA before treatment, and among them 8 (15.3\%) had angiography. The majority of patients underwent open surgery ( $n=46 / 52,88.5 \%)$, while endovascular approach has been performed only in 6 patients $(11.5 \%)$.

In the group with lower limbs lesions we performed: 12 bypass grafts (41.3\%), 8 arterial sutures raffias $(27.5 \%), 4$ arterial ligations (13.7\%), 3 coil embolization (10.3\%), 1 arterial thrombectomy (3.4\%) and 1 thigh amputation (3.4\%). We performed more frequently graft reconstruction because direct suture was not possible due to unhealthy arterial tissue. PTFE bypass was the most used technique since the ipsilateral saphenous was often injured and the harvest of the contralateral saphenous vein would have required more operating time in patients in critical conditions. In the group with lesions of upper limbs we carried out 10 direct suture (50.0\%), 4 bypass grafts (20.0\%), 2 coil embolization (10.0\%), 1 arterial ligation (5.0\%), 1 amputation (5.0\%), 1 arterial 
thrombectomy $(5.0 \%)$. One patient $(5.0 \%)$ died before reaching operating room because of associated lesions. In the group of patients with injuries in both (upper and lower) limbs, multiple direct sutures have been performed, associated in 1 patient to embolization procedure (Tab. 4).

The total length of stay in the hospital was $34 \pm 31$ days because the majority of patients suffered multiple traumas. The overall post-operative mortality rate was 5.7\% (3 patients). Two patients died because of Multiple Organ Failure, 1 died because of cerebral hemorrhage three days after limb revascularization. Among survivors we reported 5 major amputations of the lower limbs, 1 in the operating room, 2 after bypass infections and 2 after graft failure, 7 days and 32 days respectively after the first attempt of limb salvage.

\section{DISCUSSION}

Arterial traumas of the peripheral arteries of the extremities are a rare observation in patients admitted to the emergency department. Among civilians, ER evaluations are prevalently related to road accidents, criminal attacks and, less frequently, to self-harming behaviors, work and or sport traumatisms. [2,5]

As stated above, most patients with blunt trauma were poly-traumatized. In these cases, patients' life is the first priority, followed by limb salvage. The outcome in these patients is strictly influenced by co-morbidities and associated lesions. Multiple trauma patients are expected to present a higher risk of mortality than those with single injuries, being the overall mortality of multiple lesions significantly higher than mortality rates of single lesions. [6] Literature reports an association with bony injury in $35.1 \%$ of cases, nerve injury in $7.6 \%$, and injuries affecting the head, chest, or abdomen in 3.6\%. [7]

Regarding vascular injuries of limbs, it is important to differentiate minor arterial trauma, due to low speed agents, from major arterial trauma. In the first case, neither active bleeding is detected at CTA scan nor ischemia at physical examination. Therapeutic chooses usually consists in monitoring the patient during follow up, by means of physical examination and ultrasound scan. In case of major arterial trauma, with active bleeding and/or ischemia, the patient needs to be treated in the emergency room with basic and advanced life support.

Diagnostic imaging provides a valid support in identifying vascular injuries. Nowadays, delays in diagnosis and treatment are usually uncommon in patients with multiple injuries, due to the introduction of total-body CT scan, which allows a complete screening of severely injured patients with blunt multiple-trauma, determining whether surgical or angiographic intervention is needed. $[7,8]$ 
204 After diagnosis, a critical aspect in managing vascular injuries of limbs is constituted by treatment 205 decision-making process.

206 Data regarding the management of complex extremity trauma lesions are conflicting and randomized controlled studies are not reported. The absence of Level 1 and 2 evidence studies is not surprising because of the complexity of the topic. In fact, there are frequently concomitant injuries, multiple traumas and heterogenicity of treatment. Moreover, the relative rarity of such lesions contributes to complicate the analysis and to reach a consensus on their treatment.

211 For decades the classic approach to vascular lesions of extremities has been open surgery, though the growing interest in endovascular treatment has developed in the last years. $[9,10]$ Piffaretti et al[11], reported treatment of lesions amenable to endovascular repair in 10 cases over $81(12.0 \%)$ of arterial trauma lesions, represented mainly by pseudoaneurysms, dissections and arterio-venous fistulas with only one case of expanding hematoma. Immediate success was obtained in all procedures.

217 Desai et al [12], in a 8 years retrospective analysis, reported 28 endovascular repair in $21(75.0 \%)$ penetrating injuries and $7(25.0 \%)$ blunt trauma including pseudoaneurysm, extravasations, occlusions and arteriovenous fistulas, without any further distinction about localization. Patients were selected for endovascular treatment in case of no pulsatile bleeding from the wound and a suitable access site with a lesion amenable to stent graft repair at preoperative imaging, a suitable access site, and available imaging indicated a lesion amenable to stent graft repair. They reported a $100 \%$ of immediate technical success with the use of covered stent, a mean length of stay of $18 \pm$ 22.9 days. The overall limb salvage rate was $92 \%$ at 45 days and $79 \%$ at 93 days. Ganapathy et al collected 68 patients with 70 total arterial injuries. Endovascular approach was performed in 20 patients. Compared to open repair $(n=50)$ endovascular less commonly required fasciotomy ( $15 \%$ vs. $46 \%, \mathrm{p}=0.03)$ and transfusion (50\% vs. $77 \%, \mathrm{p}=0.06)$. Analysed outcomes between groups were trending higher in the endovascular group with respect to limb salvage rates at discharge (94\% vs. 89\%), median length of stay (14days vs. 9), and median follow-up (288days vs. 92) compared to the open group, but the data were not statistically significant. There was increasing utilization of endovascular repair over time (7\% of total procedures in 2009; $50 \%$ in 2014).[4] Tresson et al[13] reported endovascular treatment limited to the embolization of pelvic arteries in a series of 20 patients injured after Paris Terrorist Attack in 2015. They did not report the use of covered stents because of the devastating wounds associated, requiring always a surgical exploration.

In our cohort, endovascular treatment has been limited to 6 patients (11.5\%). Nearly $80 \%$ of our patients were either hemodynamically unstable or had multiple vessels injured on arrival to the ER. 
238 These conditions have made endovascular repair less suitable, limiting its use only in case of 239 pseudoaneurysms [14] and/or arteriovenous fistulas, though rather uncommon in civilian trauma 240 patients.

241 Despite all efforts, the prognosis of patients with arterial trauma is influenced by several factors, 242 first and foremost by the degree of general impairment evaluated using ISS; second by the kind of 243 trauma. Blunt trauma has a poorest prognosis compared to penetrating injuries that have an 244 amputation rate three times greater Amputation rate is at least three times greater than penetrating 245 injury.[15]. In our cohort, we have reported 6 cases of amputation, 5 among survivors and 1 in a 246 deceased patient, with, in most cases, lesions of the popliteal artery. Furthermore, independent risk 247 factors for amputation, including occluded graft, combined above- and below-knee injury, 248 compartmental syndrome, arterial transection, lack of intra or immediately postoperative 249 anticoagulation and associated compound fracture, play a fundamental role in the clinical 250 course. $[5,16]$

251 In our study, as reported previously (see: Results section), we had 5 major amputations of the lower 252 limbs: 1 in the OR (operating room) for a devastating lesion above the popliteal artery, 2 after graft 253 infections with septic embolism and 2 after bypass failure for the lack of postoperative anticoagulation because of concomitant brain bleeding.

\section{CONCLUSIONS}

$\underline{\text { In our experience, surgical treatment of peripheral vascular injuries is always a challenging issue, }}$ especially in complicated multiple trauma patients.

260 Although we are all, acknowledge aware of the revolutionary impact of the endovascular approach 261 in many fields, open surgery still has a role in the management of vascular trauma of the extremities 262 mainly in patients with severe concomitant lesions.

263 In these cases, the high frequency of devastating injuries and the frequent need for orthopaedic 264 and/or neurological repair, make endovascular surgery scarcely suitable.

265 On the contrary, endovascular surgery may result fundamental for the treatment of penetrating 266 injuries in areas of difficult anatomic access, such as the abdominal and pelvic area, the shoulder 267 and the neck or, in case of more peripheral lesions whose treatment can be postponed.[11,17]

268 To avoid any misleading generalization on the issue of arterial trauma repair, we strongly believe 269 that, for each patient, it is necessary to consider the anatomical locations of the vascular injury and 270 the degree of general impairment. Moreover, a multidisciplinary approach is essential in treating 271 these complex patients. Cooperation among different surgical specialists (vascular, orthopedic, 
thoracic and neurosurgeons) and interventional radiologists is the key to obtain the best clinical results.

\section{REFERENCES}

[1] Compton C. Peripheral Vascular Trauma. Perspect Vasc Surg Endovasc Ther 2005;17:297-307.

[2] Martin LC, McKenney MG, Sosa JL, Ginzburg E, Puente I, Sleeman D, et al. Management of lower extremity arterial trauma. J Trauma 1994;37:591-8.

[3] Miller-Thomas MM, West OC, Cohen AM. Diagnosing Traumatic Arterial Injury in the Extremities with CT Angiography: Pearls and Pitfalls. RadioGraphics 2005;25:S133-42.

[4] Ganapathy A, Khouqeer AF, Todd SR, Mills JL, Gilani R. Endovascular management for peripheral arterial trauma: The new norm? Injury 2017;48:1025-30.

[5] Hafez HM, Woolgar J, Robbs J V. Lower extremity arterial injury: results of 550 cases and review of risk factors associated with limb loss. J Vasc Surg 2001;33:1212-9.

[6] Rau C-S, Wu S-C, Kuo P-J, Chen Y-C, Chien P-C, Hsieh H-Y, et al. Polytrauma Defined by the New Berlin Definition: A Validation Test Based on Propensity-Score Matching Approach. Int J Environ Res Public Health 2017;14:1045.

[7] Scaglione M, Pinto A, Pedrosa I, Sparano A, Romano L. Multi-detector row computed tomography and blunt chest trauma. Eur J Radiol 2008;65:377-88.

[8] Dreizin D, Munera F. Blunt Polytrauma: Evaluation with 64-Section Whole-Body CT Angiography. RadioGraphics 2012;32:609-31.

[9] Reuben BC, Whitten MG, Sarfati M, Kraiss LW. Increasing use of endovascular therapy in acute arterial injuries: Analysis of the National Trauma Data Bank. J Vasc Surg 2007;46:1222-6.

[10] Worni M, Scarborough JE, Gandhi M, Pietrobon R, Shortell CK. Use of Endovascular Therapy for Peripheral Arterial Lesions: An Analysis of the National Trauma Data Bank From 2007 to 2009. Ann Vasc Surg 2013;27:299-305.

[11] Piffaretti G, Tozzi M, Lomazzi C, Rivolta N, Caronno R, Laganà D, et al. Endovascular treatment for traumatic injuries of the peripheral arteries following blunt trauma. Injury 2007;38:1091-7.

[12] Desai SS, DuBose JJ, Parham CS, Charlton-Ouw KM, Valdes J, Estrera AL, et al. Outcomes after endovascular repair of arterial trauma. J Vasc Surg 2014;60:1309-14. 
[13] Tresson P, Touma J, Gaudric J, Pellenc Q, Le Roux M, Pierret C, et al. Management of Vascular Trauma during the Paris Terrorist Attack of November 13, 2015. Ann Vasc Surg 2017;40:44-9.

[14] Domanin M, Castronovo LE, Fossati A, Romagnoli S, Gabrielli L. Endovascular management of post-traumatic peroneal pseudoaneurysm associated to arteriovenous fistula after sport-related injury. J Traumatol du Sport 2017;34:114-8.

[15] Rozycki GS, Tremblay LN, Feliciano D V., McClelland WB. Blunt Vascular Trauma in the Extremity: Diagnosis, Management, and Outcome. J Trauma Inj Infect Crit Care 2003;55:814-24.

[16] Guerrero A, Gibson K, Kralovich KA, Pipinos I, Agnostopolous P, Carter Y, et al. Limb loss following lower extremity arterial trauma: What can be done proactively? Injury 2002;33:765-9.

[17] Urgnani F, Lerut P, Da Rocha M, Adriani D, Leon F, Riambau V. Endovascular treatment of acute traumatic thoracic aortic injuries: A retrospective analysis of 20 cases. J Thorac Cardiovasc Surg 2009;138:1129-38. 
Figure 1

Figure 1

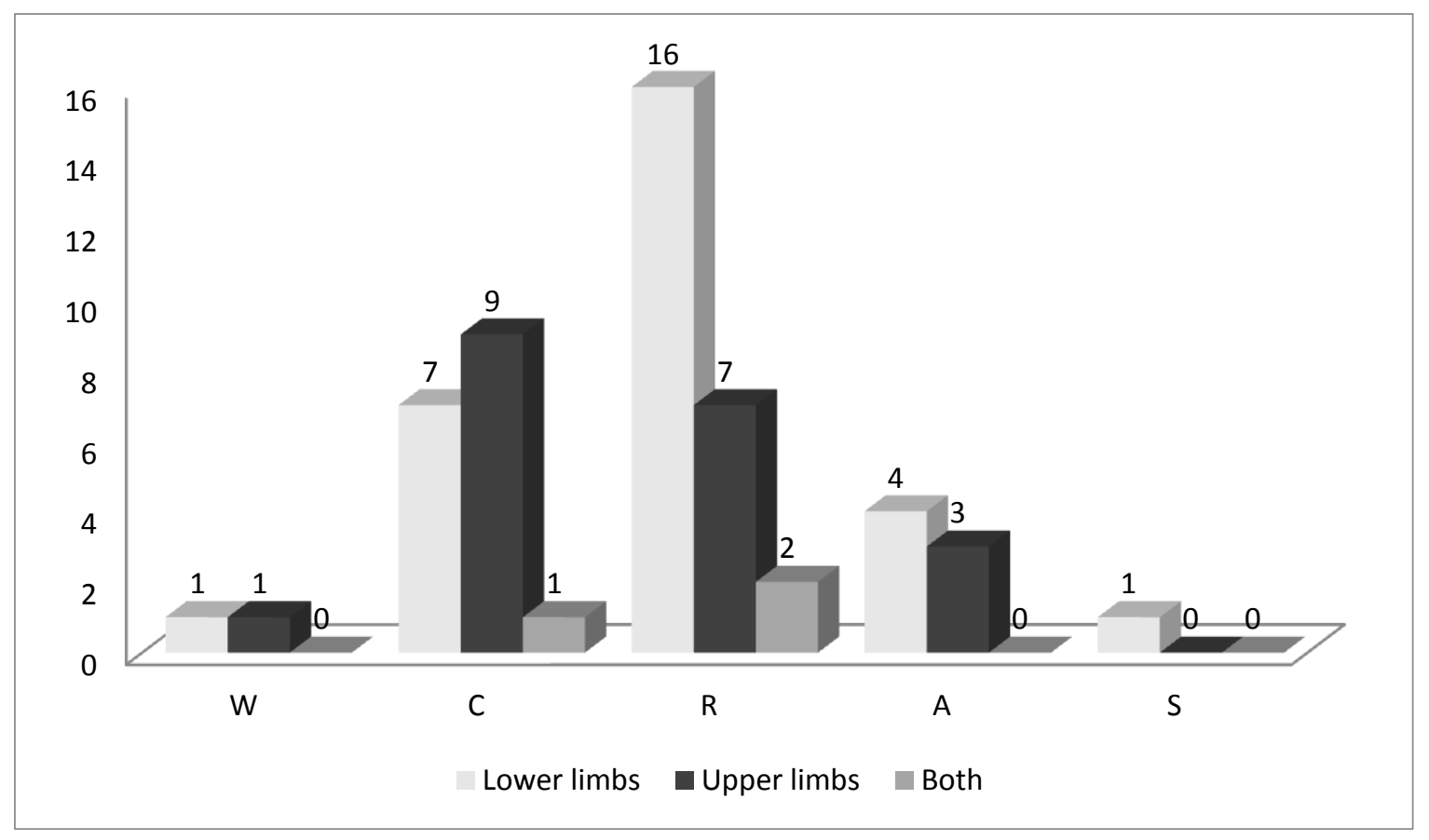





\section{Tables}

Tab. 1

\begin{tabular}{|l|l|}
\hline \multicolumn{2}{|l|}{ PARAMETERS ON THE SCENE AND AT THE ARRIVAL IN THE EMERGENCY DEPARTMENT } \\
\hline SBPs & $109 \pm 37 \mathrm{mmHg}$ \\
HRs & $89 \pm 28 \mathrm{bpm}$ \\
GCSs & $13 \pm 3$ \\
SBPed & $101 \pm 45 \mathrm{mmHg}$ \\
HRed & $94 \pm 34 \mathrm{bpm}$ \\
GCSed & $11 \pm 5$ \\
Lactates & $4 \pm 3 \mathrm{mmoll} / 1$ \\
ISS & $22 \pm 18$ \\
Need of emercengy surgery & $47(90.3 \%)$ \\
\hline
\end{tabular}

Tab. 2

\begin{tabular}{|l|l|l|l|}
\hline VASCULAR INJURY & NUMBER OF PATIENTS & ASSOCIATED LESIONS & N (\%) \\
\hline LOWER LIMBS & 29 & $\begin{array}{l}\text { orthopedic lesions } \\
\text { neurological lesions } \\
\text { venous lesions }\end{array}$ & $\begin{array}{l}17(58.6 \%) \\
6(20.6 \%) \\
6(20.6 \%)\end{array}$ \\
\hline UPPER LIMBS & 20 & $\begin{array}{l}\text { orthopedic lesions } \\
\text { neurological lesions } \\
\text { venous lesions }\end{array}$ & $\begin{array}{l}12(60.0 \%) \\
6(30.0 \%) \\
2(10.0 \%)\end{array}$ \\
\hline BOTH & & orthopedic lesions & $2(66.6 \%)$ \\
& & neurological lesions & $1(33.3 \%)$ \\
& & venous lesions & $1(33.3 \%)$ \\
\hline
\end{tabular}


Tab. 3

\begin{tabular}{|c|c|c|c|}
\hline VASCULAR INJURY & NUMBER OF PATIENTS & ARTERY INJURED & $\mathbf{N}(\%)$ \\
\hline LOWER LIMBS & 29 & $\begin{array}{l}\text { Common femoral artery } \\
\text { Superfial femoral artery } \\
\text { Profunda femoris artery } \\
\text { Popliteal artery } \\
\text { Anterior tibial artery } \\
\text { Posterior tibial artery } \\
\text { Peroneal artery } \\
\text { Common femoral artery and anterior tibial } \\
\text { artery }\end{array}$ & $\begin{array}{l}3(10.3 \%) \\
9(31 \%) \\
4(13.7 \%) \\
6(30 \%) \\
3(10.3 \%) \\
2(6.8 \%) \\
1(3.4 \%) \\
1(3.4 \%)\end{array}$ \\
\hline UPPER LIMBS & 20 & $\begin{array}{l}\text { Axillary artery } \\
\text { Brachial artery } \\
\text { Radial artery } \\
\text { Ulnar artery } \\
\text { Radial and Ulnar artery }\end{array}$ & $\begin{array}{l}3(15.0 \%) \\
9(45.0 \%) \\
5(25.0 \%) \\
1(5.0 \%) \\
2(10.0 \%)\end{array}$ \\
\hline ВОТН & 3 & $\begin{array}{l}\text { Common femoral artery and brachial artery } \\
\text { Common femoral artery and radial artery }\end{array}$ & $\begin{array}{l}1(33.3 \%) \\
2(66.6 \%)\end{array}$ \\
\hline
\end{tabular}


Tab. 4

\begin{tabular}{|c|c|c|c|}
\hline & $\begin{array}{l}\text { NUMBER OF } \\
\text { PATIENTS } \\
\end{array}$ & KIND OF TREATMENT & $\mathbf{N}(\%)$ \\
\hline LOWER LIMBS & 29 & 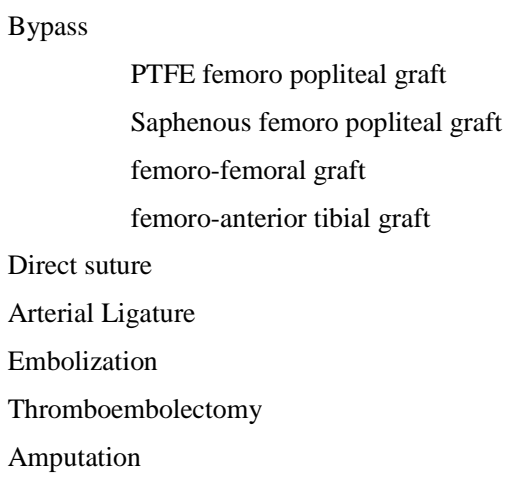 & 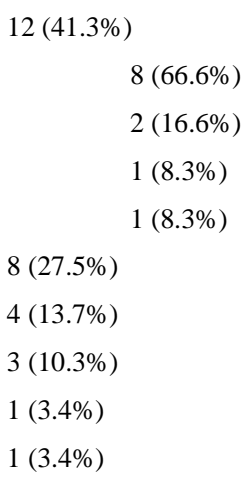 \\
\hline UPPER LIMBS & 20 & $\begin{array}{l}\begin{array}{l}\text { Direct suture } \\
\text { Bypass } \\
\qquad \text { omero-radial } \\
\text { omero-omeral }\end{array} \\
\text { Embolization } \\
\text { Arterial Ligature } \\
\text { Amputation } \\
\text { Thromboembolectomy } \\
\text { No procedure (death) }\end{array}$ & $\begin{array}{ll}10(50 \%) & \\
4(20 \%) & \\
& 2(50 \%) \\
& 2(50 \%) \\
2(10 \%) & \\
1(5 \%) & \\
1(5 \%) & \\
1(5 \%) & \\
1(5 \%) & \end{array}$ \\
\hline ВОТН & 3 & $\begin{array}{l}\text { Direct suture } \\
\text { Direct suture and embolization }\end{array}$ & $\begin{array}{l}2(66.6 \%) \\
1(33.3 \%)\end{array}$ \\
\hline
\end{tabular}

